\title{
Redox revolutions can be detected and dated through the genetic record
}

\section{CARA MAGNABOSCO}

\section{ETH Zurich}

Presenting Author: cara.magnabosco@erdw.ethz.ch

Traditionally, the biogeochemical information preserved in the rock record has been used to study the environmental conditions of Earth's past. There is however another important record of Earth's history that is only just beginning to be explored: the genomes of contemporary organisms (i.e. the genetic record). The genetic record is an under-utilized tool for studying Earth History and making predictions about the prevalence of life in the universe (and related biosignatures). In this presentation, we will describe a framework for accessing and interpreting the genetic record to learn more about past environments. Using the Great Oxidation Event (GOE) as a preliminary case study, we'll show how a combination of genetic and metabolic features can inform the timing and occurrence of the GOE. This approach utilises information regarding how changes in the environment reorganise metabolic interactions. By linking environmental change to the co-evolution of enzymes and metabolic networks, we attempt to build a model of planetary and organismal coevolution and hypothesize what metabolism on other planets might look like. 